\title{
Combined de novo and genome guided assembly and annotation of the Pinus patula juvenile shoot transcriptome
}

\author{
Erik A. Visser ${ }^{1}$, Jill L. Wegrzyn², Emma T. Steenkmap ${ }^{3}$, Alexander A. Myburg ${ }^{1}$ and Sanushka Naidoo ${ }^{\text {* }}$
}

\begin{abstract}
Background: Pines are the most important tree species to the international forestry industry, covering $42 \%$ of the global industrial forest plantation area. One of the most pressing threats to cultivation of some pine species is the pitch canker fungus, Fusarium circinatum, which can have devastating effects in both the field and nursery. Investigation of the Pinus-F. circinatum host-pathogen interaction is crucial for development of effective disease management strategies. As with many non-model organisms, investigation of host-pathogen interactions in pine species is hampered by limited genomic resources. This was partially alleviated through release of the $22 \mathrm{Gbp}$ Pinus taeda v1.01 genome sequence (http://pinegenome.org/pinerefseg/) in 2014. Despite the fact that the fragmented state of the genome may hamper comprehensive transcriptome analysis, it is possible to leverage the inherent redundancy resulting from deep RNA sequencing with Illumina short reads to assemble transcripts in the absence of a completed reference sequence. These data can then be integrated with available genomic data to produce a comprehensive transcriptome resource. The aim of this study was to provide a foundation for gene expression analysis of disease response mechanisms in Pinus patula through transcriptome assembly.

Results: Eighteen de novo and two reference based assemblies were produced for $P$. patula shoot tissue. For this purpose three transcriptome assemblers, Trinity, Velvet/OASES and SOAPdenovo-Trans, were used to maximise diversity and completeness of assembled transcripts. Redundancy in the assembly was reduced using the EvidentialGene pipeline. The resulting 52 Mb P. patula v1.0 shoot transcriptome consists of 52112 unigenes, $60 \%$ of which could be functionally annotated.

Conclusions: The assembled transcriptome will serve as a major genomic resource for future investigation of $P$. patula and represents the largest gene catalogue produced to date for this species. Furthermore, this assembly can help detect gene-based genetic markers for P. patula and the comparative assembly workflow could be applied to generate similar resources for other non-model species.
\end{abstract}

Keywords: Pinus patula, De novo transcriptome assembly, Genome guided transcriptome assembly, RNA-seq

\section{Background}

Pinus species play keystone ecological roles, representing the major component of many forests across all continents [1]. These species are also the predominantly planted trees in the global commercial forestry sector [2]. One of the largest threats to global pine forestry is the pitch canker fungus Fusarium circinatum, especially

\footnotetext{
* Correspondence: sanushka.naidoo@up.ac.za

${ }^{1}$ Department of Genetics, Forestry and Agricultural Biotechnology Institute (FABI), Genomics Research Institute (GRI), University of Pretoria, Private bag X20, Pretoria 0028, South Africa

Full list of author information is available at the end of the article
}

where susceptible Pinus species are cultivated [3]. Consequent losses caused by this fungus have large economic impacts on commercial forestry [3, 4]. Resistance to F. circinatum varies among Pinus species [5]. Species such as Pinus patula and $P$. radiata, both of which are important plantation species in southern Africa, are highly susceptible, while species such as $P$. tecunumanii are more resistant [5]. Very little is known regarding the interaction between F. circinatum and its Pinus hosts at the molecular level. Investigation of the different responses employed by susceptible and resistant hosts, such as $P$. patula and $P$. tecunumanii [5], will improve 
our knowledge of responses necessary for effective defence against $F$. circinatum.

RNA sequencing (RNA-seq) approaches have opened the way for transcriptome-wide analysis of gene expression [6]. Accurate quantification of gene expression using RNA-seq, however, requires a high quality reference sequence for read mapping. For organisms with well characterised reference genomes, such as Arabidopsis, this requirement is easily met, while organisms lacking a well characterized reference sequence present numerous challenges. Although the P. taeda v1.01 draft genome assembly is available [7], the size and fragmented state of the assembly can limit comprehensive transcriptome analysis $[8,9]$. De novo transcriptome assembly can be used to provide a reference sequence for RNA-seq analysis while circumventing potential issues arising from problems in a genome assembly [10]. De novo transcriptome assemblies are available through GenBank and the TreeGenes database [11, 12] for at least ten Pinus species (P. banksiana [13], P. contorta [13], P. lambertiana, P. massoniana, P. monticola [14], P. palustris, P. pinaster, $P$. radiata, $P$. sylvestris [15], and $P$. taeda), at various levels of completion. Of these, the $P$. taeda transcriptome is the most comprehensive, consisting of data obtained from many different tissues and developmental stages (Mockaitis et al. unpublished).

The aim of this study was to generate a resource for transcriptome profiling in P. patula by assembling the shoot transcriptome of this economically important species. We report a $P$. patula shoot transcriptome containing 52112 transcripts, of which $30844(60 \%)$ are annotated. This is the largest gene catalogue for $P$. patula to date and a major genomic resource, which will facilitate functional genomics research in this tropical pine species.

\section{Methods}

Plant material

Six month old $P$. patula seedlings, from a single open pollinated family, were sourced from Top Crop Nursery, South Africa. Seedlings were transferred to, and maintained for the duration of the trial in an environmentally controlled glasshouse at $25-28{ }^{\circ} \mathrm{C}$ without supplemental lighting and allowed to acclimatize for two weeks. F. circinatum isolate FCC3579 was cultured on $1 / 2$ strength potato dextrose agar ( $1 / 2$ PDA; Merck) after which spores were harvested by washing with $15 \%(v / v)$ sterile glycerol. Spore concentration was quantified using a haemocytometer and diluted to $5 \times 10^{4}$ spores $/ \mathrm{mL}$ by addition of $15 \%(v / v)$ sterile glycerol. Seedlings were inoculated by clipping the apical bud and pipetting $10 \mu \mathrm{L}$ of diluted spore solution onto the wound [16]. Seedlings inoculated with $10 \mu \mathrm{L}$ sterile $15 \%$ glycerol served as mock-inoculated control. Shoot tissue was harvested one day post inoculation (dpi) for three biological replicates per group (inoculated and mock-inoculated). Each biological replicate consisted of the top $4 \mathrm{~cm}$ of shoot tissue, measured from the wounded apical bud, harvested from 16 seedlings and pooled prior to being frozen using liquid nitrogen. Frozen tissue was stored at $-80{ }^{\circ} \mathrm{C}$ until use. Disease development was monitored for six weeks post inoculation by measuring lesion and stem length from the wounded apical bud on 52 plants per group. F. circinatum infection was confirmed based on culture morphology on $1 / 2$ PDA by re-isolation using tissue harvested from inoculated plants $14 \mathrm{dpi}$.

\section{RNA isolation and sequencing}

Frozen samples were homogenised using a high speed grinder (IKA-Werke, Staufen, Germany) and total RNA extracted using a modified version of Lewinsohn's protocol [17]. Modifications were as follows: All solutions were prepared using diethylpyrolecarbonate (DEPC) treated water. Approximately $5 \mathrm{~g}$ homogenised shoot tissue was placed in a $50 \mathrm{~mL}$ conical tube containing $150 \mathrm{mg}$ PVP-360 and $300 \mathrm{mg}$ PVPP before adding $15 \mathrm{~mL}$ chilled extraction buffer. Tubes were mixed by vortexing, snap frozen in liquid nitrogen and allowed to thaw on ice. Polysaccharides were precipitated by addition of $1 / 9^{\text {th }}$ volume $3.3 \mathrm{M}$ sodium acetate and $10 \%$ $(v / v)$ absolute ethanol. Nucleic acids were precipitated at $-20{ }^{\circ} \mathrm{C}$ for $4 \mathrm{~h}$. The pellet produced from ultracentrifugation was re-suspended in $100 \mu \mathrm{L}$ DEPC treated water and stored at $-80{ }^{\circ} \mathrm{C}$ until use. Total RNA samples were treated with RNase-free DNaseI enzyme (Qiagen Inc, Valencia, CA) to digest genomic DNA and purified using the RNeasy ${ }^{\circ}$ MinElute kit (Qiagen) according to the manufacturer's instructions. Concentration and integrity of purified RNA samples were evaluated using a Bio-Rad Experion $^{\text {TM }}$ automated electrophoresis system (Bio-Rad Laboratories, Hercules, CA, USA).

High quality RNA samples (RNA Integrity Number $>$ 7.5) for both groups were sequenced using Illumina HighSeq 2000 instruments (200 bp insert size, 101PE sequencing, 40 million reads per sample; BGI, Hong Kong). Sequence quality of raw RNA-seq data was assessed using FastQC v0.10.1 [18]. Quality trimming and filtering of data was performed using Sickle v1.210 [19] and all unpaired reads were discarded. Short reads ( $<40 \mathrm{bp}$ ) were removed from the filtered RNA-seq reads using SolexaQA LengthSort [20]. The trimmed and filtered read data for all six samples were combined, resulting in Dataset 1. FastUniq v1.1 [21] was used to reduce PCR artefacts from Dataset 1 by removing duplicate reads, resulting in Dataset 2. 


\section{Transcriptome assembly}

\section{Multiple $k$-mer de novo transcriptome assembly}

De novo transcriptome assembly was performed using three assemblers; Trinity r2013-11-10 [22], SOAPdenovoTrans v1.04 [23] and Velvet v1.2.10/ Oases v0.2.08 [24, 25]. Assembly with Trinity was performed on both datasets using default parameters [26], except min_contig_length $=350$, and repeated on Dataset 1 with the CuffFly parameter included. Trinity was applied to both Dataset 1 and 2 as Trinity allows for duplicate reads, however, SOAPdenovo-Trans and Velvet/Oases assemblers were used on Dataset 2 only, where duplicates were removed. Assembly with SOAPdenovo-Trans was performed on Dataset 2, for eight different $k$-mer lengths (23, $25,31,39,47,55,63$ and 71 ), with the parameters as follows: max_rd_len $=100$, rd_len_cutoff $=100$, avg_ins $=$ 200, reverse_seq $=0$, asm_flags $=3$, pair_num_cutoff $=3$, map_len $=35$, $-\mathrm{f}$ and $-\mathrm{F}$. Assembly with Velvet/Oases was performed on Dataset 2, for seven different $k$-mer lengths $(23,25,31,39,47,55$, and 63$)$, with the parameters as follows: default parameters for velveth; cov_cutoff $=10$, ins_length $=200$ and read_trkg = yes for velvetg; cov_cutoff $=10$, min_pair_count $=5$ and min_trans_lgth $=350$ for Oases.

\section{P. taeda v1.01 genome guided transcriptome assembly}

Trinity genome guided transcriptome assembly was performed on Datasets 1 and 2 using the P. taeda v1.01 draft genome assembly (ca. 14.4 million scaffolds) with a minimum contig length of 350 bp. GSNAP 2014-02-28 (Genomic Short-read Nucleotide Alignment Program) [27] was used to align reads to the reference genome for transcriptome assembly using the following paramters: --nofails, - -novelsplicing $=1,-$-localsplicedist $=250000$, --npaths $=20$. Transrate v0.3.1 [27] was used to calculate assembly quality metrics.

\section{Decreasing redundancy across assemblies}

The de novo and genome guided transcriptome assemblies were combined to form a redundant overassembly. The tr2aacds pipeline, from the EvidentialGene package [28], was used to reduce redundancy in the over-assembly by selecting for the 'optimal' set of assembled transcripts based on coding potential. The pipeline consists of five steps: (1) prediction of coding DNA sequence (CDS) and amino acid sequences for each transcript, (2) removal of redundant sequences based on coding potential among identical sequences, (3) substring de-replication to remove sequence fragments, (4) clustering of highly similar sequences into loci and (5) classification of transcripts as 'primary' or 'alternate' and discarding of low scoring 'drop' transcripts. The primary assembled transcripts were used for further assessments.

\section{Annotation}

Local alignments to the National Centre for Biotechnology Information (NCBI) non-redundant (nr) and plant protein databases were generated for the primary assembled transcripts from the tr2aacds pipeline using uBLAST (Edgar RC, unpublished) [29]. Parameters used for local alignments were: -evalue 1e-10, -weak_evalue 1e-4, -id 0.9, -weak_id 0.8. Local alignment sequence descriptions were used to remove non-pine origin sequences, sequences with significant alignments to prevalent fungal, bacterial, viral and insect sequences, from the assembly to produce the $P$. patula v1.0 draft transcriptome assembly. Blast2 $\mathrm{GO}^{\ominus} \mathrm{v} 2.7 .2$ [30] was used to predict protein domains through InterProScan 5 [31] as well as to perform Gene Ontology (GO) and Enzyme Code (EC) mapping. The P. patula transcriptome GO distribution was compared to the $P$. taeda v1.01 draft genome annotation using CateGOrizer [32]. Gene family memberships among species were visualized using custom scripts and Venn diagrams (http://bioinformatics.psb.ugent.be/webtools/Venn/).

\section{Identification of orthologous protein groups}

Annotated protein sequences for ten different species were retrieved from version 2.5 of the PLAZA protein database [33] and four external proteins sets, from conifer species, were also included (Table 1). The complete set of predicted $P$. patula v1.0 proteins from the assembled transcriptome were included. Each of the 15 protein sets were clustered to $90 \%$ identity within species and combined. Gene families were identified and annotated for the 442372 sequences using the approach described in [8]. Pfam domains [34] were assigned to the P. patula sequences using InterProScan 5.7 [31]. Identified gene families unique to $P$. patula with fewer than 5 members were discarded as these could result from assembly artefacts.

\section{Assembly validation}

The Core Eukaryotic Genes Mapping Approach (CEGMA) pipeline [35] as well as the Benchmarking Universal Single-Copy Orthologs (BUSCO) v1.1b1 tool [36] were used to identify putative core eukaryotic genes (CEGs) and universal single copy orthologs (USCOs) in the assembly as a measure of the completeness and contiguity. BUSCO analysis was performed using the early access plant dataset. In addition, conditional reciprocal best BLAST (CRBB) analysis of the $P$. patula draft transcriptome assembly, the $P$. taeda v1.01 gene models and the $P$. taeda draft transcriptome assembly was implemented with two different sets of reference sequences using Transrate [27]. Reference sets used were as follows: the P. taeda v1.01 predicted gene models available through the TreeGenes Database $[11,12]$ and the $87 P$. 
Table 1 Protein sets used for analysis of orthologous genes

\begin{tabular}{|c|c|c|c|}
\hline Source & Species & Total sequences & Clustered sequences $^{\mathrm{a}}$ \\
\hline \multirow[t]{10}{*}{ Protein sets from PLAZA v2.5 } & Arabidopsis thaliana & 27403 & 26465 \\
\hline & Glycine max & 46324 & 36364 \\
\hline & Oryza sativa & 41363 & 39541 \\
\hline & Physcomitrella patens & 28090 & 26072 \\
\hline & Populus trichocarpa & 40141 & 35668 \\
\hline & Ricins communis & 31009 & 30330 \\
\hline & Selaginella moellendorffii & 18384 & 16876 \\
\hline & Theobroma cacao & 28858 & 28294 \\
\hline & Vitis vinifera & 26238 & 24635 \\
\hline & Zea mays & 39172 & 34664 \\
\hline \multirow[t]{5}{*}{ External protein sets } & Amborella trichopoda & 25347 & 24643 \\
\hline & Picea abies & 22070 & 20869 \\
\hline & Picea sitchensis & 10521 & 8770 \\
\hline & Pinus patula & 52112 & 41956 \\
\hline & Pinus taeda & 50172 & 47225 \\
\hline
\end{tabular}

${ }^{\mathrm{a}}$ Proteins were clustered to $90 \%$ identity and only the longest sequence was retained for each cluster

patula protein sequences available through the NCBI and TrEMBL databases.

Sequence alignments against the $P$. taeda v1.01 draft genome assembly were generated to compare transcript to genome mapping of the $P$. patula v1.0 transcriptome assembly to that of other Pinus transcriptomes. Comparative alignments were produced using transcriptome data for seven other Pinus spp. available from the TreeGenes database [11, 12]: $P$. taeda (83 285 sequences), P. banksiana (21 675), $P$. contorta (14 375), P. pinaster (14 130), P. palustris (14 228), $P$. lambertiana (48 891), and $P$. radiata (4 742). Transcript sequences were aligned to the reference genome using GMAP 2014-02-28 (Genomic Mapping and Alignment Program) [37] with the following parameters: - -intronlength $=350000$, - -no-chimeras, --canonical-mode $=1, \quad-$-cross-species. The '-cross-species' parameter was excluded for alignment of the $P$. taeda transcriptome. Sequence alignments were examined at two different cut-offs, the first (95\% identity, $95 \%$ coverage) to compare mapping between species and the second (95\% identity, $50 \%$ coverage) to account for possible effects due to genome fragmentation. The $P$. patula v1.0 transcriptome assembly was further validated by alignment to fulllength Sanger sequenced $P$. taeda cDNA reference sequences present in NCBI and obtained through the TreeGenes database [11]. The 188 cDNA sequences were clustered to $90 \%$ identity. CRBB analysis to the $P$. patula v1.0 transcriptome was performed using Transrate [27].

\section{Differential expression analysis}

RNA-seq read mapping to the $P$. patula v1.0 transcriptome and expression quantification was performed through RSEM v1.2.23 (RNA-Seq by ExpectationMaximum) [38] using Bowtie2 v2.2.5 [39]. Differential expression testing was performed with EBSeq v1.10.0 [40] using median normalization (FDR < 0.05).

\section{Results and discussion}

Data generation and pre-processing

Due to the expected size of the P. patula genome (ca. 22 Gb) [41], sequencing and assembly of the genome would be a costly and challenging endeavour. Therefore, transcriptome assembly was employed to generate a $P$. patula reference sequence. RNA-seq of shoot tissue harvested $1 \mathrm{dpi}$ for inoculated and mock-inoculated samples yielded between 21 and 43 million read pairs per sample and a total of $c a .440$ million reads (Table 2). Quality filtering removed $c a .13 \%$ of reads and duplicate filtering removed a further $35 \%$ of reads. Thus Dataset 1 consisted of $c a .36 \mathrm{~Gb}$ of sequence data and Dataset 2 consisted of ca. $23 \mathrm{~Gb}$ that passed through quality filtering and were subsequently used for transcriptome assembly.

\section{Comparison of assembler output}

The completeness and quality of an assembled transcriptome is affected by the assembly program used as well as the assembly parameters used [42-48]. Comparative studies have also shown that the effectiveness of assembly programs can vary by input data set, with no assembler consistently outperforming any other [42, 43]. Due 
Table 2 Quality statistics for RNA sequencing data

\begin{tabular}{|c|c|c|c|c|c|}
\hline Data Set & Sample Name & Total Reads & Length (nt) & Q30 $(\%)$ & Total (Gb) \\
\hline \multirow[t]{7}{*}{ Raw Data } & Mock-inoculated 1 & 78344666 & 100 & 87.1 & 7.83 \\
\hline & Mock-Inoculated 2 & 86178906 & 100 & 87.1 & 8.62 \\
\hline & Mock-Inoculated 3 & 82271364 & 100 & 87.1 & 8.23 \\
\hline & Inoculated 1 & 41756756 & 100 & 86.8 & 4.18 \\
\hline & Inoculated 2 & 71697894 & 100 & 87.1 & 7.17 \\
\hline & Inoculated 3 & 80588142 & 100 & 87.1 & 8.06 \\
\hline & Total & 440837728 & & & 44.08 \\
\hline \multirow[t]{6}{*}{ Pre-processed Data } & Mock-inoculated 1 & 68237135 & $41-100$ & 100.0 & 6.45 \\
\hline & Mock-Inoculated 2 & 75081850 & $41-100$ & 100.0 & 7.10 \\
\hline & Mock-Inoculated 3 & 71683022 & $41-100$ & 100.0 & 6.77 \\
\hline & Inoculated 1 & 36270688 & $41-100$ & 100.0 & 3.43 \\
\hline & Inoculated 2 & 62484245 & $41-100$ & 100.0 & 5.90 \\
\hline & Inoculated 3 & 70235351 & $41-100$ & 100.0 & 6.64 \\
\hline Dataset 1 & Total & 383992290 & $41-100$ & 100.0 & 36.29 \\
\hline Dataset 2 & Total & 248994870 & $41-100$ & 100.0 & 23.53 \\
\hline
\end{tabular}

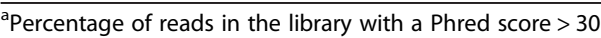

to this variability among assembler outputs, each variant assembly is likely to contain more accurate and complete assemblies at different loci. Therefore, in an effort to maximise diversity of assembled transcripts, we produced 18 de novo and two genome guided transcriptome assemblies using; Trinity, SOAPdenovo-Trans and Velvet/Oases. As expected from previous studies, large variation in the number, length and redundancy of contigs assembled was observed within and between assemblers (Fig. 1).

Trinity exhibited the most uniformity among assemblies compared to the variation among assemblies from Velvet/Oases and SOAPdenovo-Trans. An inverse relationship has been shown to exist between the number of contigs assembled and $k$-mer length used for assembly [43]. Therefore, the greater uniformity in assembled contig number between Trinity assemblies can likely be attributed to the program's implementation of a fixed $k$ mer length for all assemblies. For each value of $k$ used in assembly, SOAPdenovo-Trans resulted in the highest number of assembled contigs followed by Velvet/Oases and lastly, Trinity. In a comparative study, Trinity consistently assembled more contigs than Velvet/Oases and Trinity assemblies consistently had a higher N50 statistic [45]. Although the present study used newer versions of Velvet/Oases and Trinity, the difference in trends obtained illustrates the difference in performance of assemblers under different conditions, supporting the need for use of multiple assemblers during transcriptome reconstruction.

Trinity genome guided assemblies displayed lower GC ratios, as well as fewer predicted open reading frames
(ORFs) compared to other assemblies (Fig. 1). This was ascribed to fragmentation of the $P$. taeda v1.01 genome. Nevertheless, the two genome guided Trinity assemblies were included in downstream analysis. In total, 3447 807 assembled transcripts were used as input for the EvidentialGene tr2aacds pipeline.

The EvidentialGene pipeline selects the 'best' transcripts based on coding potential, thus selecting for the best ORFs assembled. Open reading frames were successfully predicted for ca. 2.7 million (77 \%) of the input transcripts. Of these, $49 \%$ were classified as redundant and $51 \%$ were classified as differing in CDS (non-redundant). A further $55 \%$ of non-redundant sequences were classified as perfect fragments of other longer CDS, leaving $23 \%$ of the predicted 2.7 million CDS as informative. Of the informative CDS, 60 \% were assigned to the 'drop' category and discarded. Overall, this brought about a 14-fold reduction in assembled transcript number, with only $7 \%$ of the original input sequences maintained. The resulting merged assembly contained 247035 transcripts grouped into 66377 predicted loci (Additional file 1: Table S1). This assembly was compared to the average assembly statistics across assemblies for each assembly program respectively (Fig. 2; Additional file 2: Table S2). Despite the decrease in transcript number, the proportion of transcripts containing a predicted ORF in the merged assembly was $10-40 \%$ higher compared to the average ORFs per assembly for all three assemblers. This indicates that a higher proportion of transcripts in the merged set have been accurately assembled to near-full or full length. The average length among the 1000 longest predicted proteins in the merged assembly was 1425 


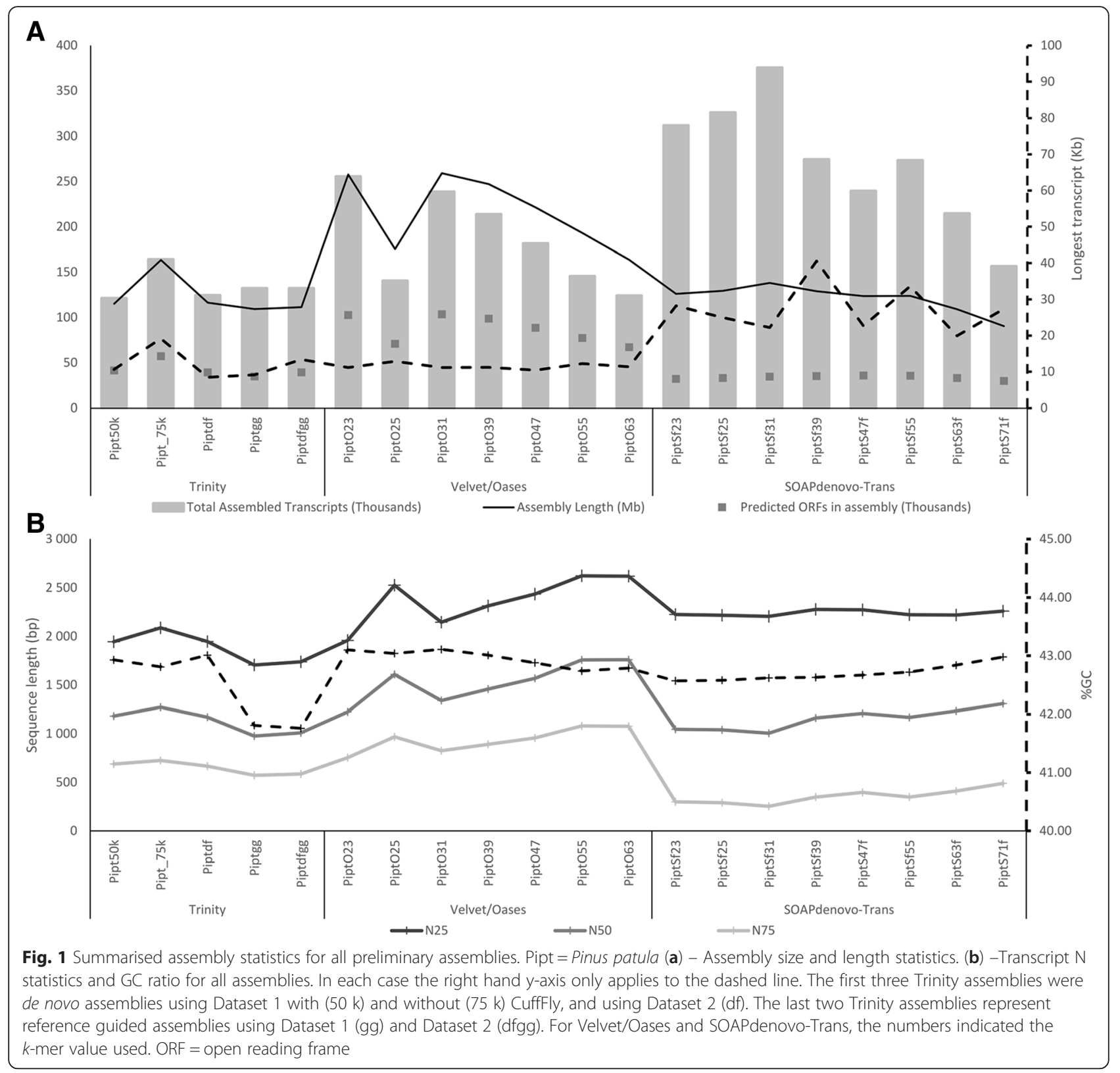

amino acids. Due to the high resource expenditure required to produce these long proteins they are often well-conserved and a biological maximum has been observed for their average length [28]. For plants this maximum observed average is ca. 1500 amino acids (based on the average between Arabidopsis, banana, cacao and poplar) [28]. This indicates that these proteins are well assembled in the merged assembly, although there is room for improvement.

\section{Annotation}

Sequence homology searches against the NR and plant protein databases successfully obtained significant local alignments for 32416 loci. Of these, 14 255 were classified as non-pine origin transcripts and filtered from the transcriptome assembly. The majority of non-pine origin transcripts $(71.62 \%)$ aligned to transcripts from Fusarium species (Additional file 3: Table S3), with most aligning to either F. fujikuroi (4 698 transcripts) or $F$. oxysporum (3 290 transcripts). A further $22.81 \%$ of nonpine origin transcripts aligned to Bipolaris maydis (1 309 transcripts), Pyrenophora tritici-repentis (992 transcripts) and Leptosphaeria maculans (949 transcripts), while the remaining $5.57 \%$ (798 transcripts) aligned to 146 different species.

Removal of non-pine origin transcripts resulted in 52 112 putative loci, classified as the $P$. patula v1.0 shoot transcriptome. The current estimates for conifer gene 


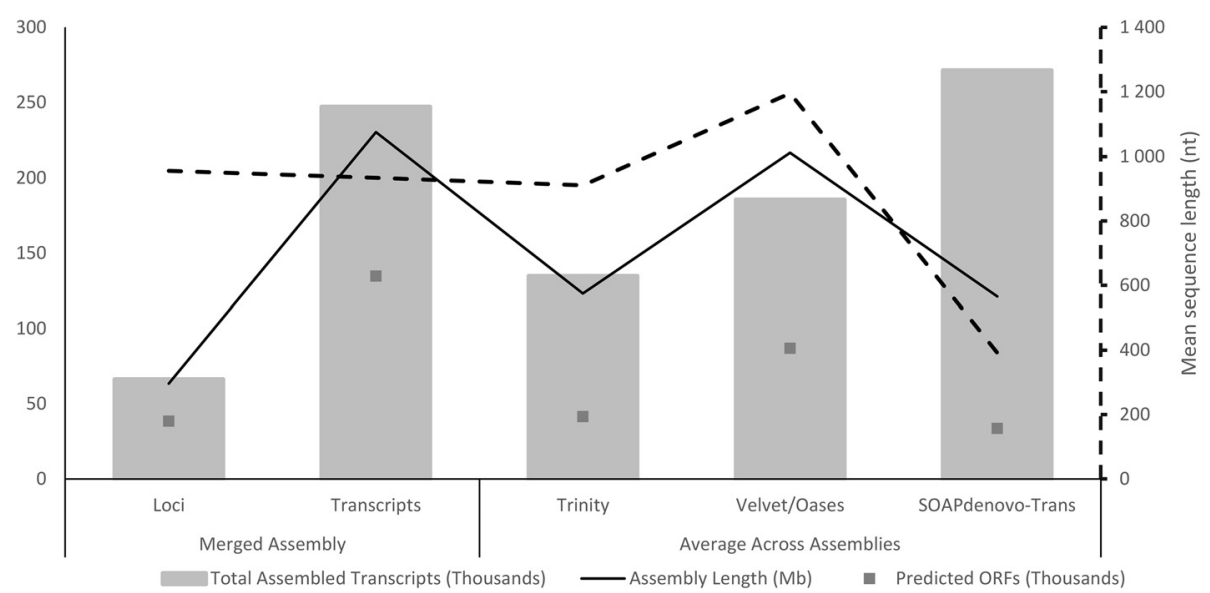

Fig. 2 Assembly statistics for tr2aacds pipeline merged assembly compared to average assembly statistics for each assembler. Assembly size and length statistics. The dashed $y$-axis only applies to the dashed line. Unfiltered output assemblies from Trinity, Velvet/Oases and SOAPdenovo-Trans were used

numbers lie between ca. 32000 for Picea glauca [49] and ca. 50000 for P. taeda and P. pinaster [8, 50]. Roughly $60 \%$ of assembled $P$. patula transcript sequences were successfully annotated, representing a wide array of molecular functions, biological processes and cellular compartment GO terms. The remaining $40 \%$ of the assembled transcript sequences could not be annotated through similarity searches, however, each sequence contained an ORF predicted by the EvidentialGene pipeline and could potentially be expressed. Thus, these sequences were not removed from the assembly as they could represent uncharacterised or conifer specific genes. The top molecular function terms for $P$. patula v1.0 transcriptome were protein binding, transferase activity and nucleic acid binding (Additional file 4: Figure S1), similar to what has been observed for P. taeda, $P$. glauca and Picea mariana $[8,51]$.

\section{Identification of putative NB-ARC defence related gene families}

Orthologous protein groups were identified by comparing 41956 clustered $P$. patula protein sequences from the assembled transcriptome to the 400416 clustered protein sequences from 14 other plant species [52] (Table 1). Tribe-MCL analysis [53] resulted in 21,492 unique gene families, with an average of 18 members per family (Additional file 5: Table S4). Gene families were identified for 396 684 (89.6\%) sequences and ranged in size from 6258 members from 15 species to 2 members from one species. Genes from the P. patula v1.0 transcriptome assembly initially clustered to 9677 gene families (35 433 genes). This was reduced to 8743 families (33 367 genes) by removing $P$. patula specific gene families with less than 5 members. While there are likely valid families in the removed set, these families were removed as most are likely to have arisen due to the remaining heterozygosity in the assembly. Of the total gene families, 2165 were unique to conifers (Fig. 3). Although this is higher than the 1554 reported by the $P$. taeda genome project $[7,8]$, it is a similar increase from the 1021 reported by the $P$. abies genome project [54]. Included in the conifer-specific gene families are 130 that were unique to $P$. patula. The largest family identified in P. patula (1 794 members) contained leucine rich repeat (LRR), toll/interleukin-1 receptor (TIR), nucleotide binding domain with an ARC motif (NB-ARC), golgi transport complex 5 (COG5) and poxvirus A32 protein motifs. This gene family was also one of the largest observed for P. taeda and had low representation among the angiosperms while representation in the moss species differed. In total, $35 \mathrm{NB}$-ARC families were identified, of which 13 were present in conifers. NBARC gene families with higher representation of angiosperm genes had little to no representation from the conifers and vice versa (Fig. 4). The NB-ARC family of genes are associated with disease resistance as the majority of resistance proteins ( $\mathrm{R}$ proteins) characterized are members of the NB-ARC and NB-LRR families [55]. Thus this difference could result from divergent $\mathrm{R}$ gene evolution between the plant lineages.

\section{Assembly validation}

Many well established metrics exist for assessment of genome assembly quality, the majority of which are based on size, such as contig and N50 size. Size based metrics such as N50 have been used in the past as a measure of transcriptome completeness [56], yet these metrics have no real biological relevance and are ineffective without prior knowledge of the actual size distribution in the sequenced data set. These metrics are also 


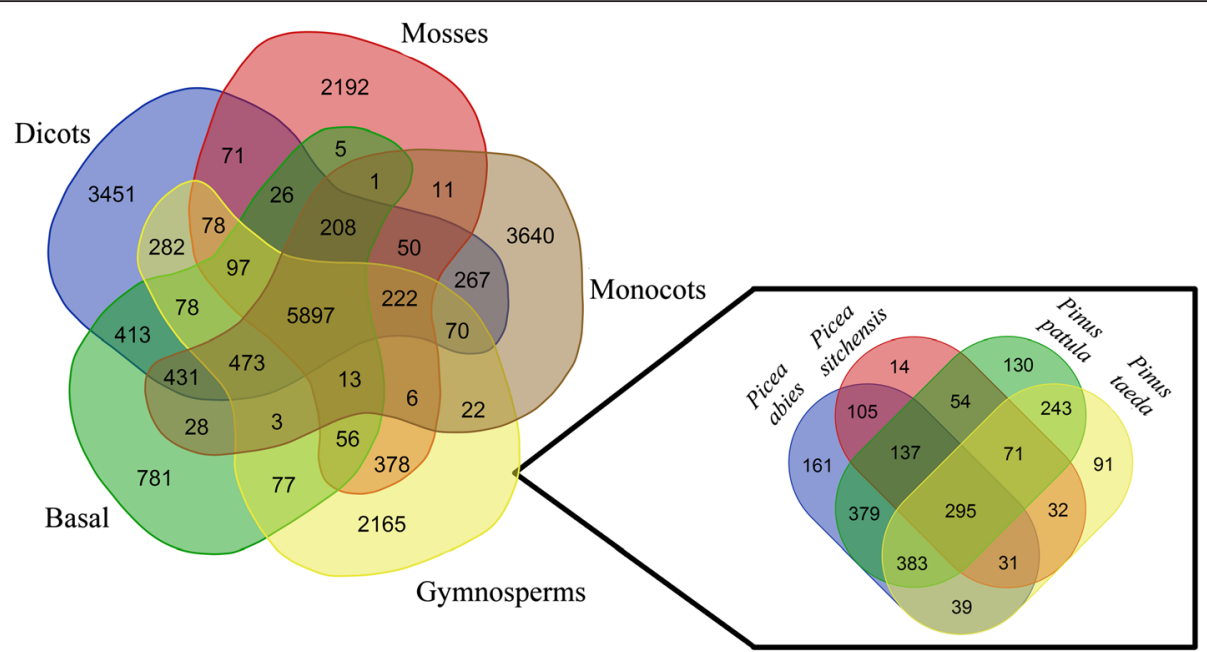

Fig. 3 Unique orthologous protein groups identified through Tribe-MCL analysis. Left Comparison of protein family counts for all identified orthologous protein groups between five different plant classifications. Right Comparison of conifer specific protein counts between four conifer species. Dicots = Arabidopsis thaliana, Glycine max, Populus trichocarpa, Ricinus communis, Theobroma cacao, Vitis vinifera. Mosses = Selaginella moellendorffii, Physcomitrella patens. Monocots = Oryza sativa, Zea mays. Gymnosperms = Picea abies, Picea sitchensis, Pinus patula, Pinus taeda . Basal = Amborella trichopoda

highly sensitive to assembly parameters and assembled isoforms (Fig. 1), which can bias quality assessment. For this reason, three reference based metrics were used to assess the transcriptome assembly; completeness, contiguity and accuracy [10]. Completeness and contiguity are closely linked. Completeness is the percentage of a reference set that has been assembled. Contiguity is the percentage of assembled reference sequences covered to $\mathrm{X} \%$, where $\mathrm{X}$ is an arbitrary minimum threshold [56]. In this study, contiguity and completeness of the P. patula transcriptome assembly was measured by comparison against four data sets.

Comparison to the CEGMA core eukaryotic proteins identified 217 (88\% completeness) of the 248 core genes, whereas 203 (82\% completeness) were identified in the P. taeda v1.01 genome [7]. At the same time, of the identified core genes, $91 \%$ showed full length alignments to the $P$. taeda v1.01 genome, while $93 \%$ (contiguity) of those from the $P$. patula v1.0 shoot transcriptome were full length. The higher completeness

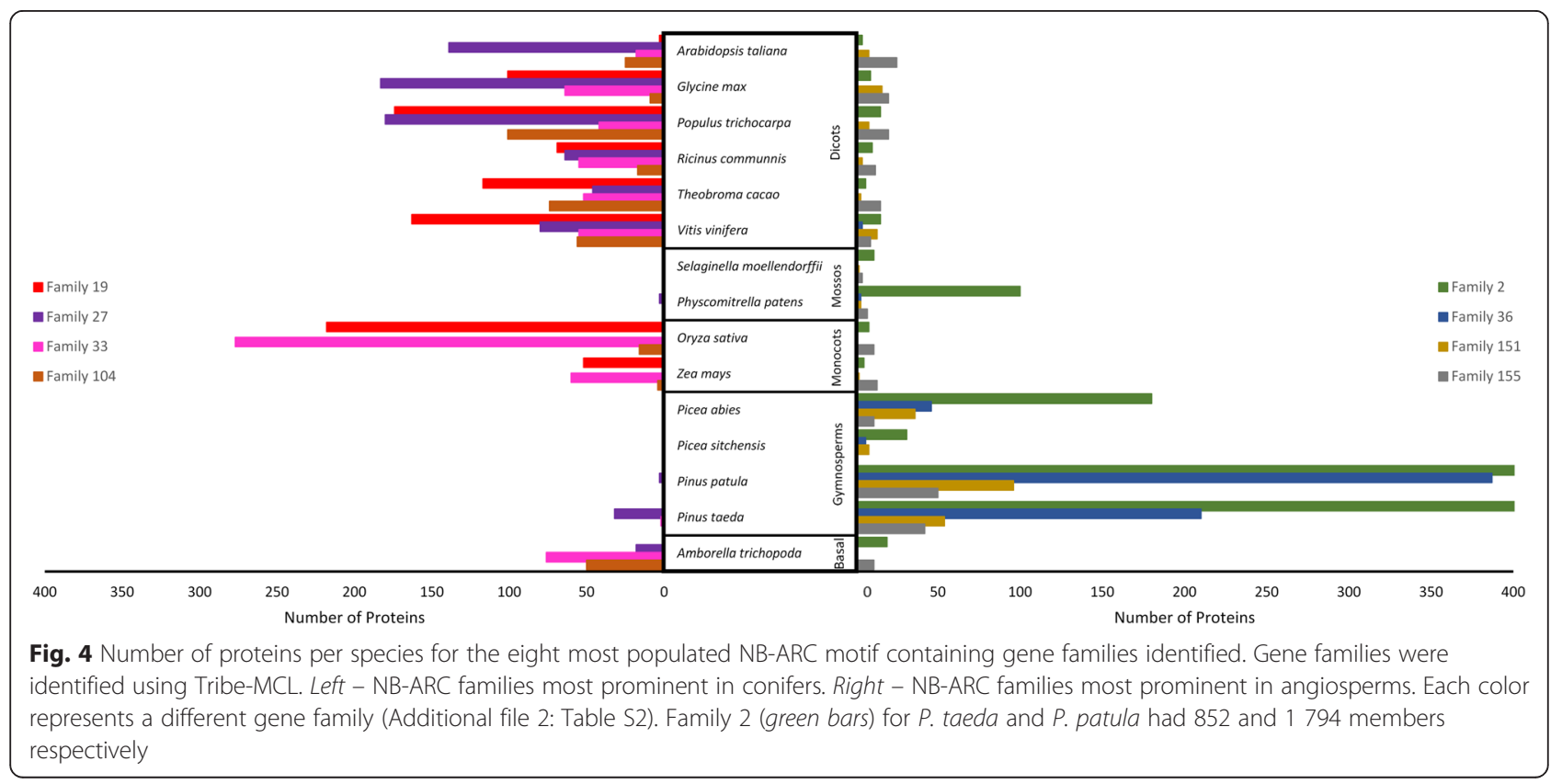


and contiguity obtained for CEGs in the P. patula transcriptome assembly compared to the $P$. taeda v1.01 genome can most likely be attributed to genome fragmentation. This illustrates the value of de novo transcriptome assembly for analysing genes missing from an incomplete genome. CEGMA analysis also identified multiple orthologs for $90 \%$ of the identified CEGs. This is likely due to the presence of high allelic variation in the data used for assembly arising from the pooled nature of the samples (pooled RNA from seedlings) used for sequencing.

BUSCO analysis against the early access plant data set identified 850 (88\%) complete BUSCOs, out of 956 groups searched, of which 307 (32\%) were duplicated. A further 26 fragmented BUSCOs were identified. The high amount of duplicated complete BUSCOs further indicate the presence of assembled haplotypes still present in the transcriptome [36].

CRBB analysis to the $P$. patula reference proteins showed a similar pattern as above when comparing completeness (49\%) and contiguity (92\%) of the P. patula transcriptome to that of the $P$. taeda transcriptome (43; $92 \%)$. This indicates higher completeness of the $P$. patula transcriptome for $P$. patula origin proteins as would be expected. The completeness (46\%) of the $P$. taeda gene models was intermediate between the transcriptomes, however, its contiguity (13\%) was notably lower. This low contiguity most likely arose due to the presence of partial genes in the high confidence gene models. The completeness and contiguity of the $P$. patula transcriptome assembly was also investigated through CRBB analysis to the P. taeda v1.0 transcriptome assembly using the gene models extracted from the $P$. taeda v1.01 genome assembly and the 87 available $P$. patula protein sequences as the reference sets. Compared to the $P$. taeda transcriptome, the $P$. patula transcriptome covered a higher proportion of the $P$. taeda gene models (at $95 \%$ coverage), had a higher proportion of reference sequences with a CRBB result and had the lowest reciprocal best hit (RBH) ratio (Table 3). Overall CRBB statistics for comparison to the $P$. patula proteins were higher for the $P$. patula transcriptome compared to both the $P$. taeda transcriptome and gene models (Table 3).

The third metric assessed was accuracy, defined as the percentage of correctly assembled bases in an assembly compared to a reference [10]. This was estimated through high-identity mapping of the assembled $P$. patula transcriptome, along with seven other pine transcriptomes, to the P. taeda v1.01 genome (Table 4). Mapping to the genome precluded calculation of completeness and contiguity, due to genome fragmentation and lack of exact gene number and location. At $95 \%$ sequence identity and query coverage thresholds a total of $64 \%$ of $P$. patula sequences mapped. The highest total mapping rates were observed for $P$. banksiana and $P$. contorta, while the lowest mapping rate was obtained for $P$. lambertiana, as expected from their phylogenetic relationship and previous studies [8]. Mapping rates obtained for the $P$. patula transcriptome were similar to

Table 3 Conditional reciprocal best BLAST (CRBB) comparisons ${ }^{a}$ of assembled Pinus patula transcripts to available Pinus taeda gene models and transcripts

\begin{tabular}{|c|c|c|c|}
\hline Query & P. taeda gene models & P. taeda v1.0 & P. patula v1.0 \\
\hline Reference & P. taeda gene models ( $n=48$ 391) & & \\
\hline Hits at $85 \%$ coverage & $99.7 \%$ & $6.6 \%$ & $8.3 \%$ \\
\hline Hits at $95 \%$ coverage & $99.7 \%$ & $3.7 \%$ & $4.2 \%$ \\
\hline Contigs with CRBB & 48363 & 29052 & 28491 \\
\hline$\%$ Contigs with CRBB & $99.9 \%$ & $34.9 \%$ & $54.7 \%$ \\
\hline References with CRBB & 48269 & 12339 & 15958 \\
\hline \% Reference CRBB & $99.7 \%$ & $25.5 \%$ & $33.0 \%$ \\
\hline Reciprocal Best Hit Ratio & 1.00 & 2.35 & 1.79 \\
\hline Reference & P. patula proteins $(n=87)$ & & \\
\hline Hits at $85 \%$ coverage & $5.8 \%$ & $39.1 \%$ & $43.7 \%$ \\
\hline Hits at $95 \%$ coverage & $2.3 \%$ & $34.5 \%$ & $40.2 \%$ \\
\hline Contigs with CRBB & 73 & 80 & 71 \\
\hline$\%$ Contigs with CRBB & $0.2 \%$ & $0.1 \%$ & $0.1 \%$ \\
\hline References with CRBB & 40 & 37 & 43 \\
\hline \% Reference CRBB & $46.0 \%$ & $42.5 \%$ & $49.4 \%$ \\
\hline Reciprocal Best Hit Ratio & 1.83 & 2.16 & 1.65 \\
\hline
\end{tabular}

${ }^{a}$ CRBB alignments for query sequences were generated against the available high confidence $P$. taeda gene models and the available $P$. patula protein sequences 
Table 4 Mapping statistics to the P. taeda v1.01 genome

\begin{tabular}{|c|c|c|c|c|c|c|}
\hline Assembly & Total Sequences & Identity & Coverage & Unique Hits & Non-unique hits & Total \% mappec \\
\hline \multirow[t]{2}{*}{ Pinus patula (EviGene Loci) } & 66377 & 95 & 95 & $33.53 \%$ & $16.78 \%$ & $50.31 \%$ \\
\hline & 66377 & 95 & 50 & $34.44 \%$ & $18.10 \%$ & $52.55 \%$ \\
\hline \multirow[t]{2}{*}{ Pinus patula v1.0 } & 52112 & 95 & 95 & $42.68 \%$ & $21.35 \%$ & $64.03 \%$ \\
\hline & 52112 & 95 & 50 & $43.84 \%$ & $23.04 \%$ & $66.87 \%$ \\
\hline \multirow[t]{2}{*}{ Pinus banksiana } & 21675 & 95 & 95 & $73.25 \%$ & $15.26 \%$ & $88.51 \%$ \\
\hline & 21675 & 95 & 50 & $74.72 \%$ & $17.62 \%$ & $92.34 \%$ \\
\hline \multirow[t]{2}{*}{ Pinus contorta } & 14375 & 95 & 95 & $70.23 \%$ & $14.91 \%$ & $85.15 \%$ \\
\hline & 14375 & 95 & 50 & $70.37 \%$ & $17.27 \%$ & $87.64 \%$ \\
\hline \multirow[t]{2}{*}{ Pinus lambertiana } & 48891 & 95 & 95 & $25.16 \%$ & $1.07 \%$ & $26.23 \%$ \\
\hline & 48891 & 95 & 50 & $31.04 \%$ & $2.07 \%$ & $33.11 \%$ \\
\hline \multirow[t]{2}{*}{ Pinus pinaster } & 14130 & 95 & 95 & $56.24 \%$ & $12.76 \%$ & $69.00 \%$ \\
\hline & 14130 & 95 & 50 & $61.27 \%$ & $16.28 \%$ & $77.54 \%$ \\
\hline \multirow[t]{2}{*}{ Pinus radiata } & 4742 & 95 & 95 & $46.06 \%$ & $11.66 \%$ & $57.72 \%$ \\
\hline & 4742 & 95 & 50 & $57.11 \%$ & $15.67 \%$ & $72.78 \%$ \\
\hline \multirow[t]{2}{*}{ Pinus sylvestris } & 11248 & 95 & 95 & $47.75 \%$ & $16.79 \%$ & $64.54 \%$ \\
\hline & 11248 & 95 & 50 & $53.90 \%$ & $20.96 \%$ & $74.87 \%$ \\
\hline \multirow[t]{2}{*}{ Pinus taeda } & 83285 & 95 & 95 & $48.72 \%$ & $7.82 \%$ & $56.54 \%$ \\
\hline & 83285 & 95 & 50 & $57.64 \%$ & $11.99 \%$ & $69.63 \%$ \\
\hline
\end{tabular}

the mapping rates obtained for the $P$. radiata and $P$. taeda transcriptome assemblies. These alignment metrics serve as a measure of transcriptome accuracy. Lowering the minimum coverage threshold to $50 \%$ increased mapping by between $2 \%$ and $15 \%$. The $P$. banksiana (3.8 \%), P. contorta (2.5\%) and P. patula (2.8\%) transcriptomes were the least affected, while the transcriptomes for $P$. radiata (15.1\%), $P$. sylvestris (10.3\%) and P. taeda (13.1\%) showed the largest increase in mapping rates, suggesting that these transcriptomes have a higher content of genes that were fragmented in the genome assembly. Comparison of accuracy metrics between assemblies should be done with care, however, as even though the $P$. taeda transcriptome showed a lower accuracy (57 \%) than P. patula, the size of the transcriptome means that it still contains ca. 10000 more accurately assembled sequences. This illustrates the importance of considering assembly size when comparing between datasets, such as the high mapping rates to the $P$. taeda v1.01 genome obtained for $P$. contorta, $P$. pinaster and $P$. radiata (Table 4). Still, more than $33000(64 \%)$ of the assembled $P$. patula sequences were shown to be accurately assembled and this number is expected to increase as fragmentation in the genome decreases.

The assembled P. patula transcripts were further compared to corresponding P. taeda complete CDS sequences to ascertain the quality of the assembly against experimentally validated data (Additional file 6: Table S5). Of the 121 cDNA sequences, 89 (73.5 \%) mapped to the P. patula transcriptome with greater than $89 \%$ identity and $80 \%$ subject coverage (Additional file 6: Table S5). Of the mapped sequences, 47 had a query coverage of more than $80 \%$ with an average sequence identity of $98.4 \pm 1.9 \%$ and an average coverage of $97.9 \pm 3.5 \%$ and $91.5 \pm 6.8 \%$ for the subject and query sequences respectively (Additional file 7: Figure S2). Thus, of the P. taeda cDNA sequences present in the assembled $P$. patula transcriptome, $52.8 \%$ have been assembled to near fulllength.

\section{Differential expression analysis}

Comparison of inoculated and mock-inoculated data sets using EBSeq identified 166 transcripts as differentially expressed between conditions (Additional file 8: Table S6). The small number of detectable differentially expressed transcripts is likely a reflection of the very early time-point investigated, where small amounts of pathogen would have been in contact with the host tissue.

Ten transcripts were up-regulated $\left(\log _{2}\right.$ (fold change) $>$ 1) in the inoculated set, relative to mock-inoculated, while 156 transcripts were down-regulated $\left(\log _{2}\right.$ (fold change $<-0.25 ; 77$ had $\log _{2}($ fold change $\left.)<-1\right)$. Among the up-regulated genes four had putative annotations (Additional file 9: Table S7). Two of these genes are involved in folate metabolism (methylenetetrahydrofolate dehydrogenase) and stomatal closure (PF03595), while the other two are linked to sugar metabolism (PREDICTED: alpha-galactosidase-like; sucrose synthase-like protein). 
In the down-regulated set 83 transcripts had putative annotations (Additional file 9: Table S7). Some of these are related to plant defence such as a putative WRKY76 encoding transcript, implicated in susceptibility against Magnoporthe oryzae but increased tolerance to cold in rice [57] and a putative phenylalanine ammonia-lyase (PAL) encoding transcript. PAL is an important enzyme for salicylic acid production and is a key enzyme in the phenylpropanoid pathway, shown to be induced in response to wounding and fungal infection in Pinus sylvestris [58]. A putative map kinase 4 is also down-regulated. In Arabidopsis, map kinase 4 is known to regulate the salicylic acid and jasmonic acid/ ethylene defence signaling [59]. Although it is tempting to speculate that the downregulation of such important transcripts in defence may, in part, contribute to susceptibility against $F$. circinatum, a detailed time-course of infection in P. patula is necessary to determine the full suite of host responses during this susceptible interaction.

\section{Conclusions}

This study presents the first transcriptome sequencing and assembly analysis for Pinus patula. The P. patula v1.0 transcriptome assembly constitutes the largest gene catalogue for this economically important species to date. More than $23 \mathrm{~Gb}$ of data was used to assemble 52 112 sequences with a total length of $52 \mathrm{Mb}$ and an average coverage of more than $200 \times$. Of these sequences, 30 844 could be assigned annotations. This transcriptome represents a major genomic resource for future studies on this tropical Pinus species, and will be used as the basis for further investigation of the host pathogen interaction between $P$. patula and $F$. circinatum. The workflow used for transcriptome assembly can in future be reapplied and altered as new sequencing data becomes available for $P$. patula to produce a more comprehensive and complete assembly. Furthermore, the workflow implemented during this study could be applied to other species where a high quality genome sequence is not available. One species to which the workflow could be applied in future is $P$. tecunumanii, a species that is closely related to P. patula [60] but which displays resistance to F. circinatum. Assembly of the P. tecunumanii transcriptome would thus allow for further investigation of the mechanisms differentiating resistance and susceptibility through comparison of defence responses in these closely related species.

\section{Availability of supporting data}

The data sets supporting the results of this article are available through the NCBI BioProject repository, [PRJNA301922; http://www.ncbi.nlm.nih.gov/bioproject/ 301922].

\section{Additional files}

Additional file 1: Table S1. EvidentialGene tr2aacds pipeline output summary. (PDF $138 \mathrm{~kb}$ )

Additional file 2: Table S2. Assembly statistics for EvidentialGene tr2aacds pipeline merged assembly compared to average statistics for each assembler. (PDF $150 \mathrm{~kb}$ )

Additional file 3: Table S3. Predicted species distribution for non-pine origin sequences removed from the Pinus patula v1.0 transcriptome. (TSV $7 \mathrm{~kb}$ )

Additional file 4: Figure S1. Molecular function gene ontology distribution for the Pinus patula v1.0 transcriptome. (TIF $713 \mathrm{~kb}$ )

Additional file 5: Table S4. Tribe-MCL gene families and annotations for all 15 species used. (CSV $1210 \mathrm{~kb}$ )

Additional file 6: Table S5. Conditional reciprocal best BLAST alignment results between full-length Sanger sequenced Pinus taeda cDNA and representative Pinus patula transcripts for each cDNA. (CSV $20 \mathrm{~kb}$ )

Additional file 7: Figure S2. Summary statistics for alignment of Pinus taeda complete CDS sequences to assembled Pinus patula transcripts. Pita $=P$. taeda. The $x$-axis represents the query $P$. taeda CDNA sequence. The solid $y$-axis (left) illustrates: cDNA query sequence length (pink circle), P. patula subject sequence length (blue square), conditional reciprocal best BLAST alignment length (gold triangle). The dashed $y$-axis (right) depicts the: percentage identity between sequences (black line), percentage coverage of the $P$. taeda cDNA by the corresponding $P$. patula transcript (green cross) and vice versa (purple plus). (TIF $2325 \mathrm{~kb}$ )

Additional file 8: Table S6. EBSeq differential expression analysis results comparing expression between inoculated and mock-inoculated data. (TSV $6517 \mathrm{~kb}$ )

Additional file 9: Table S7. Summarized list of differentially expressed genes between inoculated and mock-inoculated data with annotations. (TSV $68 \mathrm{~kb}$ )

\section{Abbreviations}

BUSCO: Benchmarking Universal Single-Copy Orthologs; ca: circa; CDS: DNA Coding Sequence; CEGMA: core eukaryotic genes mapping approach; CEGs: core eukaryotic genes; CRBB: conditional reciprocal best BLAST; DEPC: diethylpyrolecarbonate; dpi: days post inoculation; EC: enzyme code; GMAP: genomic mapping and alignment program; GO: gene ontology; GSNAP: genomic short-read nucleotide alignment program; NCBI: National Centre for Biotechnology Information; nr:

non-redundant; ORF: Open Reading Frame; PCR: polymerase chain reaction; PDA: potato dextrose agar; RBH: reciprocal best hit; RNA-seq: RNA sequencing; USCOs: Universal Single-Copy Orthologs.

\section{Competing interests}

The authors declare that they have no competing interests.

\section{Authors' contributions}

EAV performed all data generation, analysis, and prepared this manuscript. JLW provided technical support, supervision and guidance for data analysis and interpretation. ETS and AAM participated in the design of the study and edited the manuscript. SN conceived the study, provided valuable advice, direction and supervision in the planning and execution of the project and edited the final manuscript. All other technical assistance is listed in the acknowledgements.

\section{Acknowledgements}

The author would like to thank the following people for their contributions to the study: Dr Kitt Payn and Dr Nicky Jones for help in obtaining plant material and Ms Thandekile Mamni for assistance with infection trials. The author would also like to thank Forestry South Africa (for seed funding), the Genomics Research Institute (GRI) and the National Research Foundation's (NRF) Bioinformatics and Functional Genomics Programme (NBFG, UID:71255) as well as Innovation, Thuthuka and THRIP grants (Grant numbers: $84951,86936,87912)$ for financing various aspects of this study. 
Opinions expressed and conclusions arrived at, are those of the author(s) and are not necessarily to be attributed to the NRF.

\section{Author details}

'Department of Genetics, Forestry and Agricultural Biotechnology Institute (FABI), Genomics Research Institute (GRI), University of Pretoria, Private bag X20, Pretoria 0028, South Africa. ${ }^{2}$ Department of Ecology and Evolutionary Biology, University of Connecticut, Storrs, CT 06269, USA. ${ }^{3}$ Department of Microbiology and Plant Pathology, Forestry and Agricultural Biotechnology Institute (FABI), Genomics Research Institute (GRI), University of Pretoria, Private bag X20, Pretoria 0028, South Africa.

Received: 9 September 2015 Accepted: 6 December 2015 Published online: 12 December 2015

\section{References}

1. Critchfield W, Little E. Geographic distribution of pines of the world. USDA For Serv. 1966;991:1-97.

2. Indufor: Forest Stewardship Council (FSC) Strategic Review on the Future of Forest Plantations. 2012:121.

3. Wingfield MJ, Coutinho TA, Roux J, Wingfield BD. The future of exotic plantation forestry in the tropics and southern Hemisphere: Lessons from pitch canker. South Afr Forestry J. 2002;195:79-82.

4. Wingfield MJ, Hammerbacher A, Ganley RJ, Steenkamp ET, Gordon TR, Wingfield BD, et al. Pitch canker caused by Fusarium circinatum - A growing threat to pine plantations and forests worldwide. Australas Plant Pathol. 2008;37:319-34.

5. Hodge GR, Dvorak WS. Differential responses of Central American and Mexican pine species and Pinus radiata to infection by the pitch canker fungus. New For. 2000;19:241-58.

6. Wang Z, Gerstein M, Snyder M. RNA-Seq: a revolutionary tool for transcriptomics. Nat Rev Genet. 2009;10:57-63.

7. Zimin A, Stevens KA, Crepeau MW, Holtz-Morris A, Koriabine M, Marçais G, et al. Sequencing and assembly of the 22-Gb loblolly pine genome. Genetics. 2014;196:875-90.

8. Wegrzyn JL, Liechty JD, Stevens KA, Wu LS, Loopstra CA, Vasquez-Gross HA, et al. Unique features of the loblolly pine (Pinus taeda L.) megagenome revealed through sequence annotation. Genetics. 2014;196:891-909.

9. Salzberg SL, Yorke JA. Beware of mis-assembled genomes. Bioinformatics. 2005;21:4320-1.

10. Martin JA, Wang Z. Next-generation transcriptome assembly. Nat Rev Genet. 2011;12:671-82

11. Wegrzyn JL, Lee JM, Tearse BR, Neale DB. TreeGenes: A forest tree genome database. Int J Plant Genomics. 2008:2008:412875.

12. Wegrzyn JL, Main D, Figueroa B, Choi M, Yu J, Neale DB, et al. Uniform standards for genome databases in forest and fruit trees. Tree Genet Genomes. 2012:8:549-57.

13. Hall DE, Yuen MMS, Jancsik S, Quesada AL, Dullat HK, Li M, et al. Transcriptome resources and functional characterization of monoterpene synthases for two host species of the mountain pine beetle, lodgepole pine (Pinus contorta) and jack pine (Pinus banksiana). BMC Plant Biol. 2013;13:80.

14. Liu J, Sturrock RN, Benton R. Transcriptome analysis of Pinus monticola primary needles by RNA-seq provides novel insight into host resistantce to Cronartium ribicola. BMC Genomics. 2013;14:884.

15. Canales J, Bautista R, Label P, Gómez-Maldonado J, Lesur I, Fernández-Pozo $\mathrm{N}$, et al. De novo assembly of maritime pine transcriptome: implications for forest breeding and biotechnology. Plant Biotechnol J. 2014;12:286-99.

16. Porter B. Pathogenicity and competition studies on Fusarium circinatum, a pathogen of pine trees. South Africa: University of Pretoria; 2010.

17. Lewinsohn $E$, Steele $C L$, Croteau R. Simple isolation of functional RNA from woody stems of gymnosperms. Plant Mol Biol Report. 1994;12:20-5.

18. Andrews S. FastQC a quality control tool for high throughput sequence data. 2012. http://www.bioinformatics.babraham.ac.uk/projects/fastqc/.

19. Joshi NA, Fass JN: Sickle: A sliding-window, adaptive, quality-based trimming tool for FastQ. (Version 1.33) [Software] 2011, Available at https:// github.com/najoshi/sickle.

20. Cox MP, Peterson DA, Biggs PJ. SolexaQA: At-a-glance quality assessment of Illumina second-generation sequencing data. BMC Bioinformatics. 2010;11:485

21. Xu H, Luo X, Qian J, Pang X, Song J, Qian G, et al. FastUniq: A Fast De Novo Duplicates Removal Tool for Paired Short Reads. PLoS One. 2012;7:1-6.
22. Grabherr MG, Haas BJ, Yassour M, Levin JZ, Thompson DA, Amit I, et al. Trinity: reconstructing a full-length transcriptome without a genome from RNA-Seq data. Nat Biotechnol. 2013;29:644-52.

23. Xie Y, Wu G, Tang J, Luo R, Patterson J, Liu S, et al. SOAPdenovo-Trans: De novo transcriptome assembly with short RNA-Seq reads. Bioinformatics. 2014;30:1660-6.

24. Zerbino DR, Birney E. Velvet: Algorithms for de novo short read assembly using de Bruijn graphs. Genome Res. 2008;18:821-9.

25. Schulz MH, Zerbino DR, Vingron M, Birney E. Oases: Robust de novo RNAseq assembly across the dynamic range of expression levels. Bioinformatics. 2012;28:1086-92.

26. Haas BJ, Papanicolaou A, Yassour M, Grabherr M, Philip D, Bowden J, Couger MB, Eccles D, Li B, Macmanes MD, Ott M, Orvis J, Pochet N: Reference Generation and Analysis with Trinity. Volume 8; 2014

27. Smith-Unna RD, Boursnell C, Patro R, Hibberd JM, Kelly S. TransRate: reference free quality assessment of de-novo transcriptome assemblies. bioRxiv. 2015. http://dx.doi.org/10.1101/021626.

28. Gilbert D. EvidentialGene: tr2aacds, mRNA transcript assembly software. 2013. http://arthropods.eugenes.org/EvidentialGene/.

29. Edgar RC. Search and clustering orders of magnitude faster than BLAST. Bioinformatics. 2010:26:2460-1.

30. Conesa A, Götz S, García-Gómez JM, Terol J, Talón M, Robles M. Blast2GO: A universal tool for annotation, visualization and analysis in functional genomics research. Bioinformatics. 2005;21:3674-6.

31. Jones P, Binns D, Chang HY, Fraser M, Li W, McAnulla C, et al. InterProScan 5: Genome-scale protein function classification. Bioinformatics. 2014;30:1236-40.

32. Hu Z, Bao J, Reecy JM. CateGOrizer: a web-based program to batch analyse gene ontology classification categories. Online J Bioinforma. 2008;9:108-12.

33. Van Bel M, Proost S, Wischnitzki E, Movahedi S, Scheerlinck C, Van de Peer $Y$, et al. Dissecting Plant Genomes with the PLAZA Comparative Genomics Platform. Plant Physiol. 2012;158:590-600.

34. Finn RD, Bateman A, Clements J, Coggill P, Eberhardt RY, Eddy SR, et al. Pfam: The protein families database. Nucleic Acids Res. 2014;42:222-30.

35. Parra G, Bradnam K, Korf I. CEGMA: A pipeline to accurately annotate core genes in eukaryotic genomes. Bioinformatics. 2007;23:1061-7.

36. Simao FA, Waterhouse RM, loannidis P, Kriventseva EV, Zdobnov EM: BUSCO: assessing genome assembly and annotation completeness with single-copy orthologs. Bioinformatics 2015, doi:10.1093/bioinformatics/btv351

37. Wu TD, Watanabe CK. GMAP: A genomic mapping and alignment program for mRNA and EST sequences. Bioinformatics. 2005:21:1859-75.

38. Li B, Dewey CN. RSEM: accurate transcript quantification from RNA-Seq data with or without a reference genome. BMC Bioinformatics. 2011;12:232.

39. Langmead B, Salzberg SL. Fast gapped-read alignment with Bowtie2. Nat Protoc. 2012;9:357-9.

40. Leng N, Dawson JA, Thomson JA, Ruotti $\mathrm{V}$, Rissman Al, Smits BMG, et al. EBSeq: An empirical bayes hierarchical model for inference in RNA-seq experiments. Bioinformatics. 2013:29:1035-43.

41. Hall SE, Dvorak WS, Johnston JS, Price HJ, Williams CG. Flow cytometric analysis of DNA content for tropical and temperate New World pines. Ann Bot. 2000;86:1081-6

42. Duan J, Xia C, Zhao G, Jia J, Kong X. Optimizing de novo common wheat transcriptome assembly using short-read RNA-Seq data. BMC Genomics. 2012;13:392.

43. Yang $Y$, Smith SA. Optimizing de novo assembly of short-read RNA-seq data for phylogenomics. BMC Genomics. 2013;14:328.

44. Gruenheit N, Deusch O, Esser C, Becker M, Voelckel C, Lockhart PJ. Cutoffs and k-mers: Implications from a transcriptome study in allopolyploid plants. BMC Genomics. 2012;13:92

45. Clarke K, Yang Y, Marsh R, Xie LL, Zhang KK. Comparative analysis of de novo transcriptome assembly. Sci China Life Sci. 2013;56:156-62.

46. Oono $Y$, Kobayashi F, Kawahara $Y$, Yazawa $T$, Handa $H$, Itoh $T$, et al. Characterisation of the wheat (Triticum aestivum L.) transcriptome by de novo assembly for the discovery of phosphate starvation-responsive genes: gene expression in Pi-stressed wheat. BMC Genomics. 2013;14:77.

47. Steijger T, Abril JF, Engström PG, Kokocinski F, The RGASP Consortium, Hubbard TJ, et al. Assessment of transcript reconstruction methods for RNAseq. Nat Methods. 2013;10:1177-84.

48. Vijay N, Poelstra JW, Künstner A, Wolf JBW. Challenges and strategies in transcriptome assembly and differential gene expression quantification. A comprehensive in silico assessment of RNA-seq experiments. Mol Ecol. 2013;22:620-34 
49. Rigault P, Boyle B, Lepage P, Cooke JEK, Bousquet J, Mackay JJ. A white spruce gene catalog for conifer genome analyses. Plant Physiol. 2011;157:14-28.

50. Fernández-Pozo N, Canales J, Guerrero-Fernández D, Villalobos DP, DíazMoreno SM, Bautista R, et al. EuroPineDB: a high-coverage web database for maritime pine transcriptome. BMC Genomics. 2011;12:366.

51. Pavy N, Pelgas B, Laroche J, Rigault P, Isabel N, Bousquet J. A spruce gene map infers ancient plant genome reshuffling and subsequent slow evolution in the gymnosperm lineage leading to extant conifers. BMC Biol. 2012;10:84.

52. Enright AJ, Van Dongen S, Ouzounis CA. An efficient algorithm for largescale detection of protein families. Nucleic Acids Res. 2002;30(7):1575-84.

53. Van Dongen S, Abreu-Goodger C. Using MCL to Extract Clusters from Networks. In: Van Helden J, Toussaint A, Thieffry D, editors. Bacterial Molecular Networks SE - 15, Volume 804. New York: Springer; 2012. p. 281-95. Methods in Molecular Biology.

54. Nystedt B, Street NR, Wetterbom A, Zuccolo A, Lin Y-C, Scofield DG, et al. The Norway spruce genome sequence and conifer genome evolution. Nature. 2013;497:579-84.

55. Głowacki S, Macioszek V, Kononowicz A. R proteins as fundamentals of plant innate immunity. Cell Mol Biol Lett. 2011;16:1-24.

56. Martin J, Bruno VM, Fang Z, Meng X, Blow M, Zhang T, et al. Rnnotator: an automated de novo transcriptome assembly pipeline from stranded RNASeq reads. BMC Genomics. 2010;11:663.

57. Yokotani N, Sato Y, Tnabe S, Chujo T, Shimizu T, Okada K, et al. WRKY76 is a rice transcriptional repressor playing opposite roles in blast disease resistance and cold stress response. J Exp Bot. 2013;64:5085-97.

58. Adomas A, Heller G, Guosheng LI, Olson A, Tzu-Ming C, Osborne J, et al. Transcript profiling of a conifer pathosystem: response of Pinus sylvestris root tissues to pathogen (Heterobasidion annosum) invasion. Tree Physiol. 2007;27:1441-58.

59. Broderson P, Peterson M, Bjorn Nielsen H, Zhu S, Newman MA, Shokat KM, et al. Arabidopsis MAP kinase 4 regulates salicylic acid- and jasmonic acid/ ethylene-dependent responses via EDS1 and PAD4. Plant J. 2006;47:532-46.

60. Eckert AJ, Hall BD. Phylogeny, historical biogeography, and patterns of diversification for Pinus (Pinaceae): Phylogenetic tests of fossil-based hypotheses. Mol Phylogenet Evol. 2006;40:166-82.

\section{Submit your next manuscript to BioMed Central and we will help you at every step:}

- We accept pre-submission inquiries

- Our selector tool helps you to find the most relevant journal

- We provide round the clock customer support

- Convenient online submission

- Thorough peer review

- Inclusion in PubMed and all major indexing services

- Maximum visibility for your research

Submit your manuscript at www.biomedcentral.com/submit

) Biomed Central 